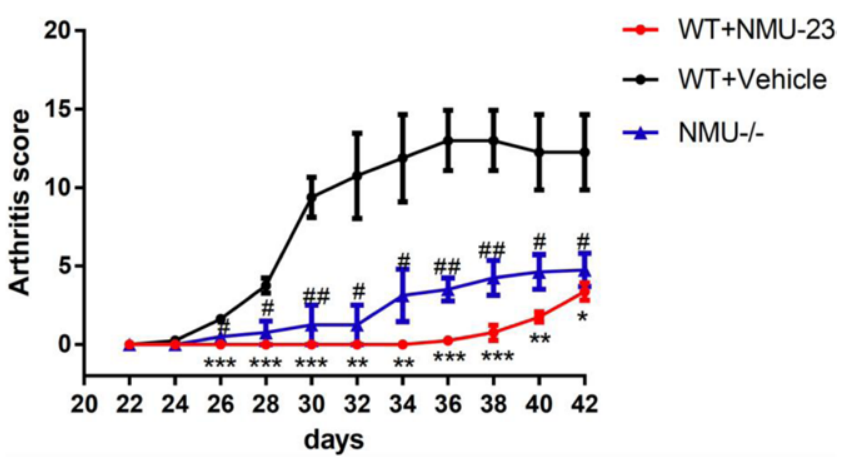

Figure 1. Arthritis score among 3 group of mice.

Disclosure of Interests: None declared

DOI: 10.1136/annrheumdis-2020-eular.5108

SATURDAY, 06 JUNE 2020

Rheumatoid arthritis - prognosis, predictors and outcome

\section{SAT0026 HIGHER LEVELS OF NATURAL ANTI- PHOSPHORYLCHOLINE ANTIBODIES ARE ASSOCIATED WITH LOWER RISK OF INCIDENT CARDIOVASCULAR EVENTS IN YOUNGER PATIENTS WITH RHEUMATOID ARTHRITIS}

S. Ajeganova ${ }^{1,2}$, M. Andersson ${ }^{3}$, J. Frostegård ${ }^{4}$, I. Hafström ${ }^{2} .{ }^{1}$ Vrije Universiteit Brussel, Brussels, Belgium; ${ }^{2}$ Karolinska Institutet, Stockolm, Sweden; ${ }^{3}$ Lund University, Department of Clinical Sciences, Rheumatology, Lund, Sweden; ${ }^{4}$ Karolinska Institutet, Institute of Environmental Medicine, Immunology and Chronic Disease, Stockolm, Sweden

Background: The increased cardiovascular (CV) risk in rheumatoid arthritis (RA), especially in seropositive RA, is not fully explained by traditional risk factors. Immuno-inflammatory mechanisms and autoantibodies could be involved in the pathogenesis of atherosclerotic disease. Recent studies have suggested that anti-phosporylcholine antibodies (anti-PC) of IgM subclass counteract the generation of senescent and IL-17+ T-cells, have atheroprotective effects and may play a role in formation and stabilization of atherosclerotic plaque.

Objectives: To investigate the association between IgM anti-PC antibodies with cardiovascular (CV) morbidity in patients with RA in age and sex groups and by serostatus.

Methods: The study population was derived from the BARFOT early RA cohort, recruited in 1994-1999. The outcome was CV events i.e. AMI, angina pectoris, coronary intervention, ischemic stroke and TIA tracked through the Swedish Hospital Discharge and the National Cause of Death Registries. The RA-disease measures and traditional risk factors were assessed according to the protocol. Sera collected at inclusion and the 2-year visit were analyzed with ELISA to determine levels of anti-PC IgM (Athera CVDefine kit, Athera Biotechnologies $A B$ ). The Kaplan-Meier estimates and Cox proportional-hazards regression models were applied. Analysis were stratified by median level of IgM anti-PC and performed within strata of age, sex and RA-autoantibodies.

Results: In all, 654 patients with early RA, $68 \%$ women, mean (SD) age 55(14.7) years, DAS28 5.2(1.3), 60\% RF-positive and 60\% ACPA-positive without prevalent CVD were included in this analysis. The level of IgM anti-PC at baseline was median (IQR) of 60.9(36.4-94.9) and at 2 years 56.0(32.3-84.2) U/ml. During follow-up of $>10$ years, 141 incident CV events $(21.6 \%)$ were registered. The levels of anti-PC both at inclusion and after 2 years of observation were lower in participants who experienced $C V$ event than in those who did not, $p=0.020$ and $p=0.012$. The $C V$ event-free survival differed between patients with levels of anti-PC above median compared with those with levels below, $p=0.003$ by log-rank test. The risk for incident $\mathrm{CV}$ event showed a 0.6 -fold hazard $(95 \% \mathrm{Cl}, 0.4-0.8)$ among patients with higher anti-PC levels as compared with those with lower levels, $p=0.003$. In the age groups, the risk for incident CV event was lower in patients aged $<55$ years at inclusion than in those who were older, hazard ratio (HR) $0.40(0.17-$ $0.94), p=0.036$. This result persisted when adjusted for sex and all traditional risk factors, HR 0.36 (0.14-0.92), $\mathrm{p}=0.032$. Also, the risk for incident $\mathrm{CV}$ events was lower in patients with higher anti-PC levels in females, HR 0.61 (0-39-0.45), and double RF- and ACPA- negative patients, 0.44 (0.21-0.90), in crude analyses. The favourable effect of anti-PC at baseline and the CV outcome was not observed in ages $>55$ years, males, ACPA+ and RF+ patients. There were no significant association between anti-PC level at 2 years and outcome.

Conclusion: These results suggest that higher levels of IgM anti-PC are associated with a lower risk of incident $\mathrm{CV}$ events over 10 years in younger patients. The favourable atheroprotective effect of $\mathrm{gM}$ anti-PC may be a part of explanation of lower risk of atherosclerotic disease in younger persons, females and in those with seronegative RA. Acknowledgments : 6th Framework Program of the European Union (grant LSHM-CT-2006-037227 CVDIMMUNE)

Disclosure of Interests: Sofia Ajeganova: None declared, Maria Andersson: None declared, Johan Frostegård Grant/research support from: Unconditional competitive grant from Amgen, related only to PCSK9, not the topic of this abstract, Ingiäld Hafström: None declared DOI: 10.1136/annrheumdis-2020-eular.3713

\section{SAT0027

SAT0027

DEVELOPMENT AND VALIDATION OF A NOMOGRAM COMBINING CLINICAL AND HISTOPATHOLOGICAL SYNOVIAL FEATURES FOR PREDICTING EARLY TREATMENT RESPONSE IN NAIVE TO TREATMENT RHEUMATOID ARTHRITIS

S. Alivernini' ${ }^{1}$, B. Tolusso' ${ }^{2}$ M. Gessi' ${ }^{2}$, M. R. Gigante ${ }^{2}$, A. Mannocci ${ }^{3}$,

L. Petricca ${ }^{2}$, S. Perniola ${ }^{2}$, C. DI Mario ${ }^{4}$, A. L. Fedele ${ }^{2}$, L. Bui ${ }^{2}$, A. Capacci ${ }^{2}$

D. Bruno ${ }^{4}$, G. La Torre ${ }^{3}$, F. Federico ${ }^{1}$, G. Ferraccioli ${ }^{4}$, E. Gremese ${ }^{1} .{ }^{1}$ Fondazione Policlinico Universitario A. Gemelli IRCCS - Università Cattolica del Sacro Cuore, Rome, Italy; ${ }^{2}$ Fondazione Policlinico Universitario A. Gemelli IRCCS, Rome, Italy; ${ }^{3}$ Sapienza University of Rome, Rome, Italy; ${ }^{4}$ Università Cattolica del Sacro Cuore, Rome, Italy

Background: Rheumatoid Arthritis (RA) is characterized by synovial tissue (ST) heterogeneity at disease onset in terms of inflammatory degree and microanatomical organization being related to treatment response.

Objectives: To develop a multiparametric tool for baseline treatment response prediction including disease characteristics and histopathologic features of ST biopsies, using a large single center (SYNGem Unit) naive to treatment RA cohort. Methods: 240 naive to treatment RA who underwent US-guided ST biopsy, at the first clinical evaluation, were enrolled. Clinical and immunological characteristics were recorded for each patient. All ST FFPE specimens were stained with H\&E and classified by a pathologist, blinded to clinical characteristics, using the Krenn score [1] to assess the degree of ST inflammation. All naive to treatment RA were treated according to the T2T scheme and DAS remission rate at 6-12 months was recorded. On the basis of the regression analysis, a nomogram was constructed that incorporated the significant factors predicting the "achievement of DAS-Remission at 6 months follow-up" in naive RA. The performance of the nomogram was assessed by discrimination and calibration.

Results: Univariate analysis showed that RA who achieved early (6 months) DAS-remission had, at baseline, significantly lower total Krenn score $(p<0.001)$, shorter symptoms duration $(p=0.005)$ and lower disease activity $(p<0.001)$ than RA not achieving this clinical outcome. ROC curve analysis revealed that RA having, at baseline, a total Krenn score <4.5 [(AUC)95\%C.I.: 0.67(0.60-0.74),p<0.001] achieved more likely DAS-remission at 6 months $(53.1 \%)$ than RA with total Krenn score $\geq 4.5(28.9 \%, \mathrm{p}<0.001)$. Interestingly, RA whose ST was biopsied within 3 months from joint symptoms beginning showed significantly lower ST inflammation as total Krenn score than RA whose ST was analyzed among 3-12 months $(p=0.04)$ or after 12 months $(p=0.002)$ since symptoms beginning. However, in terms of follicular structure presence, the microanatomical organization of the synovial inflammatory infiltrate did not differ comparing RA whose ST was biopsied within 3 months from joint symptoms beginning (44.4\%) and RA whose ST was biopsied among 3-12 months (47.6\%, $\mathrm{p}=0.74)$ or after 12 months $(52.7 \%, \mathrm{p}=0.33)$ since symptoms beginning.

Logistic regression analysis revealed that, at baseline, being VERA, not having HDA and having a total Krenn score $<4.5$ were synergistic factors of DAS-remission achievement at 6 months [OR:10.5(95\%IC:2.28-48.01);p<0.05]. Based on the regression analysis, a nomogram integrating baseline clinical (disease activity and duration) and histological (total Krenn score) characteristics was developed in which the value of each of the variables was given a point score. A total score was calculated by adding each single point score and, by projecting the value of the "total points" score to the "probability" line up to $87.5 \%$.

Conclusion: Krenn score is a reliable tool for the semi-quantitative assessment of ST inflammation on US-guided ST biopsies being contingent to baseline disease characteristics and can be integrated within a nomogram to better predict the therapeutic response in naive to treatment RA.

\section{References:}

[1] Krenn V, et al. Histopathology 2006

Disclosure of Interests: Stefano Alivernini: None declared, Barbara Tolusso: None declared, Marco Gessi: None declared, Maria Rita Gigante: None declared, Alice Mannocci: None declared, Luca Petricca: None declared, Simone Perniola: None declared, Clara Di Mario: None declared, Anna Laura Fedele: None declared, Laura Bui: None declared, Annunziata Capacci: None declared, Dario Bruno: None declared, Giuseppe La Torre: None declared, Francesco Federico: None declared, Gianfranco Ferraccioli: None declared, Elisa Gremese Speakers bureau: Abbvie, BMS, Celgene, Jannsen, Lilly, MSD, Novartis, Pfizer, Sandoz, UCB DOI: 10.1136/annrheumdis-2020-eular.6020 